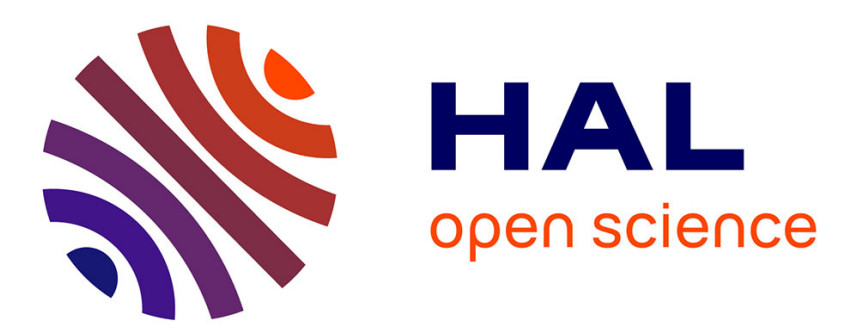

\title{
Multifidelity-CMA: a multifidelity approach for efficient personalisation of 3D cardiac electromechanical models
}

Roch Molléro, Xavier Pennec, Hervé Delingette, Alan Garny, Nicholas Ayache, Maxime Sermesant

\section{- To cite this version:}

Roch Molléro, Xavier Pennec, Hervé Delingette, Alan Garny, Nicholas Ayache, et al.. MultifidelityCMA: a multifidelity approach for efficient personalisation of 3D cardiac electromechanical models. Biomechanics and Modeling in Mechanobiology, 2017, pp.1-16. 10.1007/s10237-017-0960-0 . hal01656008

\section{HAL Id: hal-01656008 https://hal.inria.fr/hal-01656008}

Submitted on 5 Dec 2017

HAL is a multi-disciplinary open access archive for the deposit and dissemination of scientific research documents, whether they are published or not. The documents may come from teaching and research institutions in France or abroad, or from public or private research centers.
L'archive ouverte pluridisciplinaire HAL, est destinée au dépôt et à la diffusion de documents scientifiques de niveau recherche, publiés ou non, émanant des établissements d'enseignement et de recherche français ou étrangers, des laboratoires publics ou privés. 


\title{
Multifidelity-CMA: a Multifidelity Approach for Efficient Personalisation of 3D Cardiac Electromechanical Models
}

\author{
Roch Molléro - Xavier Pennec - Hervé Delingette • Alan Garny • \\ Nicholas Ayache · Maxime Sermesant
}

Received: date / Accepted: date

\begin{abstract}
Personalised computational models of the heart are of increasing interest for clinical applications due to their discriminative and predictive abilities. However, the simulation of a single heartbeat with a 3D cardiac electromechanical model can be long and computationally expensive, which makes some practical applications, such as the estimation of model parameters from clinical data (the personalisation), very slow. Here we introduce an original multifidelity approach between a 3D cardiac model and a simplified "0D" version of this model, which enables to get reliable (and extremely fast) approximations of the global behavior of the 3D model using 0D simulations. We then use this multifidelity approximation to speed-up an efficient parameter estimation algorithm, leading to a fast and computationally efficient personalisation method of the 3D model. In particular, we show results on a cohort of 121 different heart geometries and measurements. Finally, an exploitable code of the 0D model with scripts to perform parameter estimation will be released to the community.
\end{abstract}

Keywords Cardiac Electromechanical Modeling . Reduced Model · Multi-fidelity Modeling · Parameter Estimation · Finite Element Mechanical modeling

Roch Molléro

Inria, Asclepios Research Project, Sophia Antipolis, France

Tel.: (+33).6.75.66.31.89

E-mail: rochmollero@hotmail.com

Xavier Pennec $\cdot$ Hervé Delingette · Nicholas Ayache $\cdot$ Maxime Sermesant

Inria, Asclepios Research Project, Sophia Antipolis, France

E-mail: maxime.sermesant@inria.fr

Alan Garny

Auckland Bioengineering Institute, University of Auckland, Auckland, New Zealand

\section{Introduction}

Electromechanical models of the heart simulate the physical behavior of a patient's heart, in order to perform advanced analysis of the cardiac function. They are of increasing interest to help clinicians in their daily practice (Kayvanpour et al., 2015; Baillargeon et al., 2014; Smith et al., 2011). In particular, recent works have been successful in predicting haemodynamic changes in cardiac resynchronization therapy (Sermesant et al., 2012), ventricular tachycardia inducibility and dynamics (Chen et al., 2016), as well as in detecting and localising infarcts (Duchateau et al., 2016) using 3D personalised models.

After building the patient's heart mesh geometry, the simulated heartbeat has to match clinical data, such as ejected blood volume and pressure measurements, or more detailed information about regional motion and abnormalities available from imaging modalities such as 3D Echocardiography or Cine MRI. This is done by finding adequate simulations settings (boundary conditions, loading constraints) and values of model parameters such as myocardial stiffness and contractility (Xi et al., 2011; Chabiniok et al., 2012). This phase of parameter estimation is usually referred to as the personalisation of the cardiac model (Marchesseau et al., 2013b) and results in a personalised cardiac model (Wang et al., 2012) made of a patient-specific heart geometry (Schaerer et al., 2006) and patient-specific biomechanical parameters.

A wide variety of 3D computer heart models exists in the literature, which describe the anatomy and physiology of the heart at various scales. For example the 3D mesh describing the heart geometry can be made of very different numbers of nodes, and the cellular electromechanical phenomena underlying the build-up of 
myocardial forces can either be described with a large number of equations, or simplified equations. We refer to the two comprehensive reviews of (Chabiniok et al., 2016) and (Clayton et al., 2011) for a large discussion of various models of different scales, types and implementations. The scale and precision (also known as fidelity) of the model is chosen according to the study and the available data. In general, the time required to compute a simulation increases with its level of detail. The simulation of a 3D heartbeat using some of the most complex 3D models can take up to several hours of computation on computers with hundreds of cores (Panthee et al., 2016). This means that for applications where many simulations need to be repeatedly performed (e.g. parameter estimation), computational time becomes a real issue.

The joint use of low-fidelity models to approximate a high-fidelity model and lower the computational burden has been investigated by the multifidelity modeling community since (Kennedy and O'Hagan, 2000). As described in Peherstorfer et al. (2016), a modelmanagement method usually handles and feeds the outputs of a low-fidelity model (e.g. a simplified model, a regression model or a projection-based model) to an underlying application-specific method (e.g. an optimisation algorithm) as surrogates to the high-fidelity model outputs. The method also optionally decides when to recompute simulations of the high-fidelity model to guarantee the accuracy of the low-fidelity approximation.

Here we present an original 0D/3D multifidelity approach for the personalisation of $3 \mathrm{D}$ cardiac models (Fig. 1). First, from our 3D cardiac model, we derived and implemented a simplified "0D" model which is faster by 4 orders of magnitude. This was performed as proposed in Caruel et al. (2014), by approximating the geometry of the ventricle as a sphere and assuming spherical symmetry and homogeneity of the electromechanical behavior.

Then, we introduce a multifidelity coupling in order to approximate 3D model simulations from 0D model simulations. To this end, we build a parameter mapping which converts parameters of the $3 \mathrm{D}$ model into parameters of the 0D model, based on a few representative 3D simulations in the parameter space (called the sigma-simulations). Outputs of the 3D model are then approximated from 0D model simulations, thus enabling a reduction of the computational burden when a large number of 3D simulations outputs are required.

Finally, we present a multifidelity personalisation method, built by adapting an efficient optimization algorithm called CMA-ES (Hansen, 2006) to use approximations of the $3 \mathrm{D}$ simulations obtained through the multifidelity coupling instead of the real 3D simulations. This leads to a fast and computationally efficient personalisation method for the 3D model parameters.

A preliminary version of this work was described in (Mollero et al., 2016). In this manuscript, we propose a significantly extended methodology for the multifidelity coupling. First, the sigma-simulations selection is performed so that additional computational gains are possible when some estimated parameters have the same equations and values in both models. Then, a more robust, non-linear, parameter mapping is used. An additional step is finally introduced to correct the possible errors arising during the estimation of $0 \mathrm{D}$ model parameters. We also present an improved methodology for the multifidelity personalisation method which enables the use of a single coupling for many iterations of CMA-ES. This is done while simultaneously ensuring that the approximation is accurate enough for the optimisation algorithm, resulting in an overall estimation which is 5 times faster than in (Mollero et al., 2016) on average.

In terms of results, we present extended results and discussions for both the multifidelity coupling and the multifidelity personalisation method. The approximation accuracy of the coupling is compared to an hypersurface interpolation method and the personalisation method is compared to BOBYQA (Powell, 2009), a commonly used derivative-free optimization algorithm. This leads to an extended discussion on the computational aspects of our method in a parallel environment. This work is illustrated on a personalisation problem involving 5 parameters and 3 outputs, and we demonstrate results on a database of 121 different geometries and clinical values, which we believe to be one of the largest cohort of personalised cardiac cases to date. This personalisation took around 2.5 days on our cluster.

Lastly our 0D model equations are encoded in the CellML format (Cuellar et al., 2003) and made available for download from the Physiome Model Repository ${ }^{1}$ (Yu et al., 2011). Python scripts to perform parameter estimation in the $0 \mathrm{D}$ model will be released within 1 month of publication, from the same location.

\section{Multi-fidelity Cardiac Modelling and Personalisation Framework}

In this work we use both a $3 \mathrm{D}$ electromechanical model which can simulate the behaviour of complex patientspecific heart geometries, and a reduced "0D" version of this model which can be summarized in a few equations. Both models rely on the same mechanical laws

\footnotetext{
1 https://models.physiomeproject.org/e/470
} 


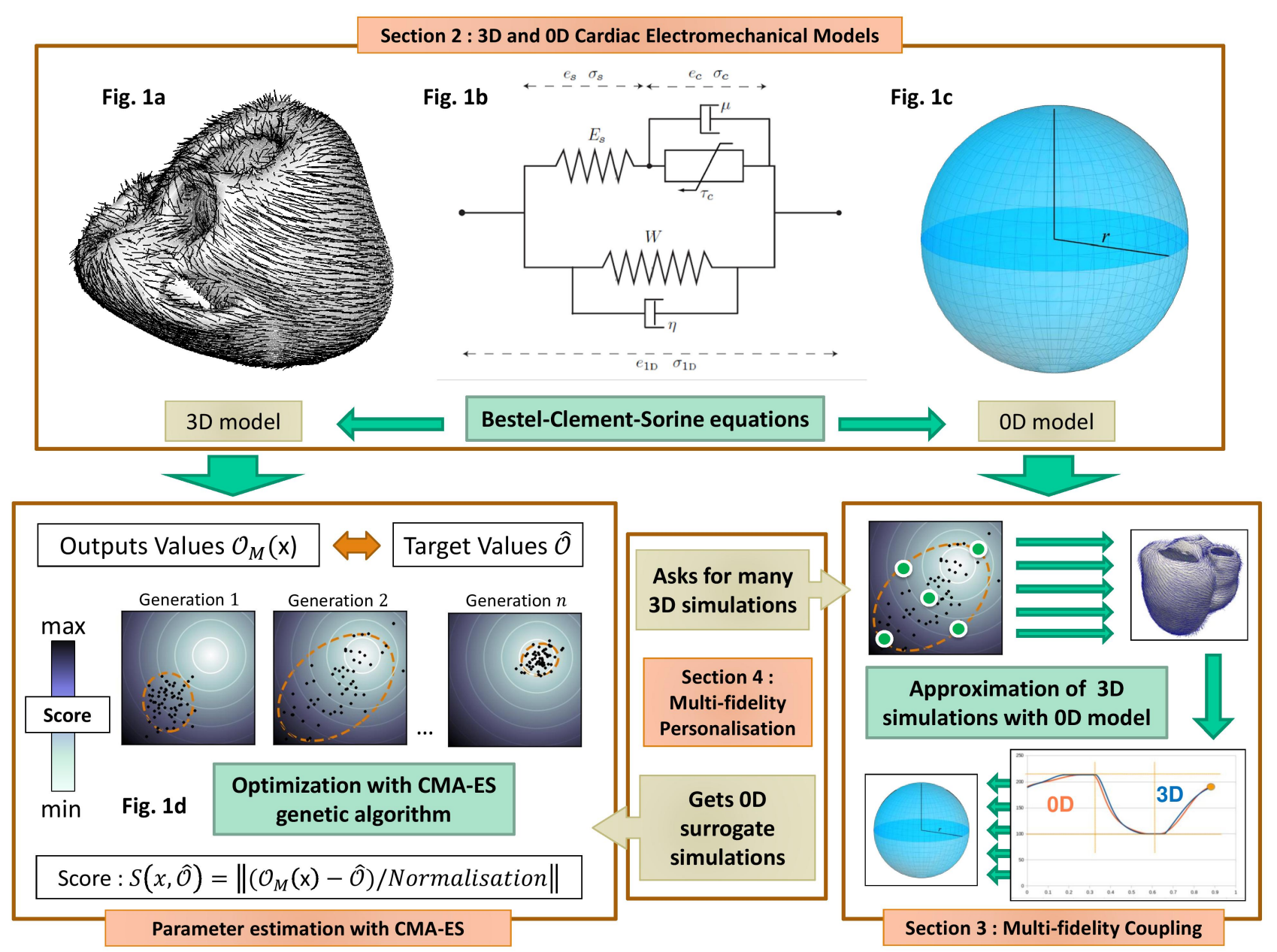

Fig. 1: 3D and 0D cardiac models (1.a, 1.b and 1.c). Our multifidelity personalisation method performs parameter estimation in the 3D model using CMA-ES (1.d), based on 0D simulations obtained through the multifidelity coupling between the models.

but simplifying assumptions are made on the geometry of the 0D model to derive its equations. We also introduce the personalisation framework for the parameters of both models.

\subsection{The 3D Cardiac Model}

Our 3D cardiac eletromechanical model is an implementation of the Bestel-Clement-Sorine (BCS) model (Chapelle et al. (2012)) by (Marchesseau et al., 2010, 2013a) in $\mathrm{SOFA}^{2}$, an open-source simulation software. The model uses the following items as an input:

- A 3D tetrahedral biventricular mesh, either synthetically created or derived from segmented MRI images.

\footnotetext{
2 www.sofa-framework.org
}

- A set of myocardial fibres directions, defined at each node of the mesh. Here we use synthetic fibres from the rule-based of Streeter (1979).

- A set of depolarisation and repolarisation times at each node of the set computed from an electrophysiology model. Here we use the Eikonal model as described in (Sermesant et al., 2012).

Myocardial forces are then computed at each node and at each time step from the equations of the BCS model. Then the myocardial motion (mesh nodes velocities) as well as ventricular volumes and pressures are computed at each time step of the cardiac cycle from these forces. See APPENDIX A for a description of the mechanical model equations and parameters. With myocardial meshes made of around 15000 nodes and a time step of $5 \mathrm{~ms}$, a single beat of $0.9 \mathrm{~s}$ takes $15 \mathrm{~min}$ utes to compute on average on a single-core (Intel(R) Core(TM) i7-4600U [2.10GHz]). 


\subsection{The 0D Cardiac Model}

As described in Caruel et al. (2014), it is possible to derive the equations of a fast $O D$ model of the heart, which relies on the same BCS equations. This is done by making the following simplifying assumptions on the geometry, the electrical activation and the properties of the material:

1. The ventricle has a spherical shape.

2. The material is incompressible.

3. The electrical activity is synchronous and homogeneous over the sphere.

With these assumptions of spherical symmetry, myocardial forces and motion are also spherically symmetric and can be entirely described by the inner radius $r$ of the ventricle. Deformation and stress tensors can also be reduced to a simple form (see Caruel et al. (2014)), which leads to a system of a dozen equations (see APPENDIX B).

We implemented the equations into $\mathrm{C}$ code and solve the system of equations using an explicit Forward Euler method with a temporal discretisation of 0.01 milliseconds. This leads to the simulation of around 15 beats per second. We also encoded the 0D model in the CellML format (Cuellar et al., 2003), which is an open standard based on the XML markup language to store and exchange computer-based mathematical models. This model can be downloaded from the Physiome Model Repository ${ }^{3}$ and easily exploited through the software OpenCOR (Garny and Hunter, 2015).

\subsection{Parameter estimation Framework for Cardiac Models}

After building the model, parameter estimation is usually the first step to analyse clinical data with a model. It consists in finding parameter values for which the simulation with the model reproduces available values and quantities in the data, such as pressure or volume measurements. In particular when the geometry is patient-specific, this phase is called cardiac model personalisation (Marchesseau et al., 2013b; Kayvanpour et al., 2015).

Formally, we consider a cardiac model $M$, a set of simulated quantities called the outputs $\mathscr{O}$ and a subset $\mathscr{P}_{\mathrm{M}}$ of varying parameters of the model (while the other parameters are supposed fixed). Given a vector of these parameters $x \in \Omega_{\mathrm{M}}$, we note $\mathscr{O}_{\mathrm{M}}(x)$ the values of the outputs $\mathscr{O}$ in the simulation of $\mathrm{M}$ with parameter values $x$. The goal of personalisation is to find parameter

\footnotetext{
3 https://models.physiomeproject.org/e/470
}

values $x * \in \Omega_{M}$ for which the outputs values $\mathscr{O}_{\mathrm{M}}(x *)$ best match some target values $\widehat{\mathscr{O}}$.

This is an inverse problem, which can be tackled by different methods (see the review of Chabiniok et al. (2016)). We propose here a parameter estimation framework (Fig 1.d) through derivative-free optimization, using an efficient genetic algorithm called CMAES (Hansen, 2006).

\subsubsection{Robust Optimisation With the Genetic Algorithm CMA-ES}

We define the score $S(x, \widehat{\mathscr{O}})$ of some parameter values $x$ as the $L_{2}$ distance between $\mathscr{O}_{\mathrm{M}}(x)$ and $\widehat{\mathscr{O}}$, normalised by the Hadamard (coordinate-by-coordinate) division $\oslash$ with a vector $\mathscr{N}$, in order to compare outputs with different units:

$S(x, \widehat{\mathscr{O}})=\left\|\left(\mathscr{O}_{\mathrm{M}}(x)-\widehat{\mathscr{O}}\right) \oslash \mathscr{N}\right\|$

We then perform a derivative-free optimisation with the genetic algorithm $C M A-E S$, which aims at minimising this score S. The algorithm (which stands for Covariance Matrix Adaptation Evolution Strategy) asks at each iteration $n$ for the scores of $m$ points $x_{i} \in \Omega_{\mathrm{M}}$ (a generation), drawn from a multivariate distribution with covariance $I_{n}^{c}$ and mean $I_{n}^{m}$. Then, it combines bayesian principles of maximum likelihood with natural gradient descent on the ranks of the points scores in the generation to update both $I_{n}^{c}$ and $I_{n}^{m}$.

The CMA-ES algorithm has many advantages in this context. First, it can explore a large and unbounded parameter space while still performing a local search at each iteration, and has shown very good results on problems involving hundreds of parameters to optimise (Geijtenbeek et al., 2013). Second, because the updates of the $I_{n}^{c}$ and $I_{n}^{m}$ only depend on the score ranks, it is very robust to outliers in the generation, in particular to parameter values for which the simulation diverges (in which case we give an arbitrary high score to these parameters).

Also, since each score comes from an independent simulation, this algorithm is well suited to parallel environments. We can either decide to set a very high population size $m$ and do many parallel simulations (in this case the algorithm can converge in a few iterations), or a lower population size and rely instead on many iterations of the algorithm for convergence.

\subsubsection{Application to the OD model}

Because the 0D model is extremely fast ( 15 beats per seconds), parameter estimation is also very fast with the $0 \mathrm{D}$ model. For example with a population size of 50 
points per generation it takes less than 50 generations and 3 minutes on a 4-core computer (with parallel computation of the simulations within each generation) to make most of the problems with sets of up to 10 outputs and parameters converge.

In our current implementation, 29 outputs can be extracted from the pressure, volume and flow curves and 25 parameters of the 0D model can be estimated. Python scripts to automatically perform the parameter estimation will be released within 1 month after the publication, available for download from the Physiome Model Repository ${ }^{4}$.

\subsubsection{Application to the 3D model}

It is possible to apply directly this framework to the $3 \mathrm{D}$ model, but the computational burden can become an issue because of the time required to compute the 3D simulations. Indeed, either we set a small population size, but we need many iterations of CMA-ES (of around 15 minutes each). Or we set a high population size but is the number of parallel CPUs used at the same time which may become prohibitive. In Section 4, our multifidelity personalisation method lowers this computational burden by replacing the outputs values of $3 \mathrm{D}$ simulations with approximations computed from 0D simulations through a multifidelity coupling between the two models, as explained in Section 3.

\section{Multi-fidelity Coupling: Approximating global outputs values of the 3D Model}

We present here a multifidelity coupling between the $3 \mathrm{D}$ and the 0D model. We will call global outputs of the models quantities which can be computed from the simulations of both models, such as the total ejected blood volume (stroke volume) or the minimal (diastolic) aortic pressure.

We consider a set of $N_{1}$ parameters of the $3 \mathrm{D}$ model $\mathscr{P}_{3 \mathrm{D}}$, a set of global outputs $\mathscr{O}$, and a set of parameter values $x_{i} \in \Omega_{3 D}$ of the parameters $\mathscr{P}_{3 \mathrm{D}}$. The goal is to get approximations of the values $\mathscr{O}_{3 \mathrm{D}}\left(x_{i}\right)$ by performing 0D simulations and only a few 3D simulations.

We will illustrate the method on the following problem: a set of 5 parameters $\mathscr{P}_{3 \mathrm{D}}$ of the $3 \mathrm{D}$ model, and a set of 3 outputs $\mathscr{O}$ listed in Table 1 . We want to approximate the output values for $m=30$ simulations with parameters $x_{i}$, drawn from a multivariate distribution (as in a CMA-ES iteration).

\footnotetext{
${ }^{4}$ https://models.physiomeproject.org/e/470
}

Table 1: Sets of 3D model parameters and global outputs in the example.

\begin{tabular}{l}
\hline Global outputs $\mathscr{O}$ \\
\hline Stroke Volume $S V$ \\
Mean Aortic Pressure $M P$ \\
Diastolic Aortic Pressure DP \\
\hline Parameters of the $3 D$ model $\mathscr{P}$ \\
\hline Contractility $\sigma$ \\
Stiffness $c_{1}$ \\
Peripheral resistance $R_{p}$ \\
Aortic Compliance $C$ \\
Central Venous Pressure $P_{\text {ve }}$
\end{tabular}

3.1 Global Strategy: Building a Mapping Between 3D and 0D Mechanical Parameters

Because they rely on the same equations, both models display many similar trends in their global outputs values when some parameters vary. For example, if a 3D simulation and a 0D simulation have the same stroke volume, the stroke volume variations with changes in the haemodynamic parameters (such as the peripheral resistance) are very similar in both models.

However some parameters do not behave exactly the same, and are not always even in the same range of values. This is especially the case of mechanical parameters such as the 3D and 0D dampings which rely on different equations. But even for parameters from the same equations in both models (such as $\sigma$ and $c_{1}$ ) the values might be very different in $0 \mathrm{D}$ and $3 \mathrm{D}$ simulations with similar outputs, due to the different assumptions.

Formally, there is no trivial function which can convert the $x_{i} \in \Omega_{3 D}$ into values $y \in \Omega_{0 D}$ of $0 \mathrm{D}$ model parameters $\mathscr{P}_{0 \mathrm{D}}$, for which the global outputs values $\mathscr{O}_{0 \mathrm{D}}(y)$ and $\mathscr{O}_{3 \mathrm{D}}(x)$ are the same (or at least close). The idea of the multifidelity coupling is to find OD model simulations which are similar to a few selected 3D simulations, then build a parameter mapping $\phi$ between the parameters of both models. We use the following strategy:

1. First we perform a few representative 3D simulations within the domain of interest (called the sigma-simulations with parameters $X_{i} \in \Omega_{3 D}$ ).

2. Then, for each $3 \mathrm{D}$ sigma-simulation with parameter values $X_{i} \in \Omega_{3 D}$, we estimate parameter values $Y_{i} \in$ $\Omega_{0 D}$ of a coupled $0 D$ simulation which approximates the outputs $\mathscr{O}$ of the $3 \mathrm{D}$ sigma-simulation .

3. From those 3D model parameters $X_{i}$ and 0D model parameters $Y_{i}$ we derive a parameter mapping $\phi$ which converts $3 \mathrm{D}$ parameters into $0 \mathrm{D}$ parameters. 


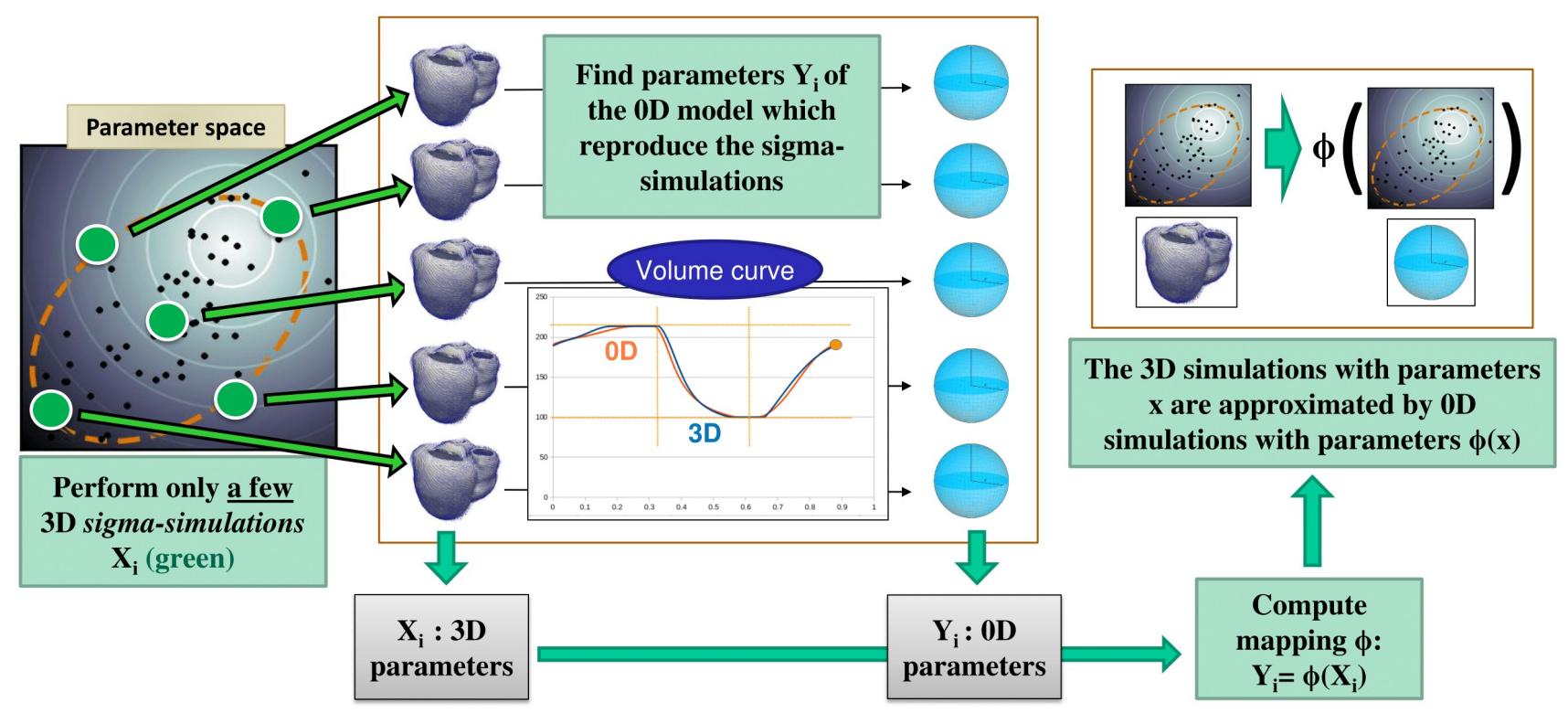

Fig. 2: Multi-fidelity Coupling: After performing a few 3D sigma-simulations, we find coupled OD simulations for each of those simulations. Then we build a parameter mapping which converts parameters of the 3D model into parameters of the 0D model, in order to approximate 3D simulations outputs with the outputs of $0 \mathrm{D}$ simulations.

4. Finally, we approximate the global outputs values $\mathscr{O}_{3 \mathrm{D}}(x)$ of all the $3 \mathrm{D}$ simulations $x_{i} \in \Omega_{3 D}$, from the 0D simulations with parameters $\phi\left(x_{i}\right) \in \Omega_{0 D}$. This is done by adding a correction term $\psi$ which is learnt, to avoid numerical errors in the previous steps.

The overall process is illustrated in Fig 2. In the sequel, we first discuss the selection of representative sigma-simulations (Sec. 3.2), then the computation of coupled OD simulations (Sec. 3.3), then the parameter mapping $\phi$ (Sec. 3.4) and the correction term $\psi$ (Sec. 3.5). Finally we give numerical results of the multifidelity approximation in Sec. 3.6.

3.2 Sigma-Simulations: Performing Representative 3D Simulations Within the Domain of Interest

We consider a subset $\mathscr{P}^{\prime}{ }_{3 \mathrm{D}} \subset \mathscr{P}_{3 \mathrm{D}}$ of $N_{2}<N_{1}$ parameters which cannot be converted directly into 0D model parameters. In order to assess the global outputs variations to these parameters in the set of $x_{i} \in \Omega_{3 D}$, we perform a few selected simulations in the domain $\Omega_{3 D}$.

To this end, we perform PCA on the set of $x_{i} \in \Omega_{3 D}$, which gives $N_{1}$ eigenvectors of the set. Then we extract the $N_{2}$ eigenvectors $\mathbf{d}_{\mathbf{k}}$ which display the maximal variations of the parameters $\mathscr{P}^{\prime}{ }_{3 \mathrm{D}}$. This is done by sorting the eigenvectors by the norm of their projection of the subspace made by the coordinates corresponding to the parameters $\mathscr{P}_{3 \mathrm{D}}^{\prime}$, and selecting the $N_{2}$ largest.

One sigma-simulation is then performed at the center $\left(X_{0}\right)$ of the domain of interest $\Omega_{3 D}$ and pairs are performed equidistant of $X_{0}$ in each of the $N_{2}$ extracted directions $\left(X_{k}^{+}=X_{0}+d_{k}\right.$ and $X_{k}^{-}=X_{0}-d_{k}$ for $\left.k=1 . . N_{2}\right)$. This gives a total of $2 N_{2}+1$ representative simulations in the domain.

In our example, the three haemodynamics parameters $R_{p}, C$ and $P_{\text {ve }}$ have the same values and the same equations in both models, so we can use the same values directly in the two models. On the other hand, the contractility $\sigma$ and the stiffness $c_{1}$ do not have the same values in both models so we need to assess how their variation is going to impact the global outputs. We then extract the $N_{2}=2$ directions for which the variations of $\sigma$ and $c_{1}$ are maximal, and perform a total of $2 N_{2}+1=5$ sigma simulations with parameters $X_{0}, X_{1}^{+}, X_{2}^{+}, X_{1}^{-}$and $X_{2}^{-}$.

\subsection{Coupled 0D Simulations: Reproducing global outputs of the 3D Sigma-Simulations with 0D Simulations}

Then for each sigma-simulation with parameters $X_{i}$, $i=1 . .2 N_{2}+1$ and output values $\mathscr{O}_{3 \mathrm{D}}\left(X_{i}\right)$, we want to find a corresponding $0 \mathrm{D}$ simulation which has similar global outputs values. To this end, we consider another set $\mathscr{O}^{\prime}$ of global outputs called the coupling outputs, 
and a set of $0 \mathrm{D}$ parameters $\mathscr{P}^{\prime}{ }_{0 \mathrm{D}}$ called the coupled $0 D$ parameters.

We then find values $Y_{i}$ of the parameters $\mathscr{P}^{\prime}{ }_{0 \mathrm{D}}$ for which the coupling outputs values $\mathscr{O}^{\prime}{ }_{0 \mathrm{D}}\left(Y_{i}\right)$ of the 0D model simulations are the closest from the coupling outputs values $\mathscr{O}^{\prime}{ }_{3 \mathrm{D}}\left(X_{i}\right)$ of the $3 \mathrm{D}$ model sigmasimulations, with all other parameters being the same in both models. This is what we call a coupled OD simulation.

This is done by performing, for each 3D sigmasimulation $k=1 . . N_{2}$, an independent parameter estimation of the $0 \mathrm{D}$ model parameters $\mathscr{P}_{0 \mathrm{D}}$ using the method presented in Section 2.3. The target values $\widehat{\mathscr{O}^{\prime}}$ for the coupling outputs $\mathscr{O}^{\prime}$ are their values in the corresponding $3 \mathrm{D}$ sigma-simulation.

In our example, since we want to approximate outputs from the volume and pressure curves (see Table 1), we need to approximate these curves with the 0D model. We then chose a set of 4 coupling outputs $\mathscr{O}^{\prime}$ from these curves, and a set of 3 coupled $0 D$ parameters $\mathscr{P}_{\mathrm{OD}}$ of the $0 \mathrm{D}$ model to estimate, both listed in Table 2.

Table 2: Coupling outputs, Coupled 3D parameters, Coupled OD parameters which are estimated and $D i$ rectly Mapped Parameters in the example of Fig 3.

\begin{tabular}{l}
\hline Coupling outputs $\mathscr{O}$ \\
\hline Maximal Volume Vmax \\
Minimal Volume Vmin \\
Mean Aortic Pressure MP \\
Diastolic Aortic Pressure DP \\
\hline Coupled $3 D$ parameters $\mathscr{P}^{\prime}{ }_{\mathrm{DD}}$ \\
\hline Contractility $\sigma$ \\
Stiffness $c_{1}$ \\
\hline Coupled $0 D$ parameters $\mathscr{P}_{\mathrm{OD}}$ \\
\hline Contractility $\sigma$ \\
Stiffness $c_{1}$ \\
Resting Radius $r_{0}$ \\
\hline Directly Mapped Parameters \\
\hline Peripheral resistance $R_{p}$ \\
Aortic Compliance $C$ \\
Central Venous Pressure $P_{\mathrm{ve}}$
\end{tabular}

After performing the 5 parameter estimations for the 5 sigma-simulations, we found 5 coupled $0 D$ simulations with parameters $Y_{i}, i=1 . .5$ which have similar coupling outputs values, which we report in Table 3 . We also display the pressure and volume curves of the $3 \mathrm{D}$ sigma-simulations and coupled $O D$ simulations in Fig 3.
Table 3: Coupling outputs values for the 3D sigmasimulations with parameters $X_{i}$ and the corresponding coupled $O D$ simulations with parameters $Y_{i}$

\begin{tabular}{c|cccc} 
& $\mathrm{Vmax}(\mathrm{ml})$ & $\mathrm{Vmin}(\mathrm{ml})$ & $\mathrm{MP}(\mathrm{Pa})$ & $\mathrm{DP}(\mathrm{Pa})$ \\
\hline$X_{0}$ & 129 & 51.8 & 10278 & 7290 \\
$X_{1}^{+}$ & 129 & 35.5 & 10034 & 7590 \\
$X_{2}^{+}$ & 129 & 64.4 & 9556 & 7614 \\
$X_{1}^{-}$ & 129 & 69.8 & 10743 & 7906 \\
$X_{2}^{-}$ & 129 & 40.4 & 10761 & 6664 \\
\hline$Y_{0}$ & 128 & 53.9 & 10318 & 7310 \\
$Y_{1}^{+}$ & 125 & 41.0 & 10107 & 7679 \\
$Y_{2}^{+}$ & 128 & 66.3 & 9591 & 7634 \\
$Y_{1}^{-}$ & 129 & 70.4 & 10759 & 7910 \\
$Y_{2}^{-}$ & 128 & 42.5 & 10806 & 6683
\end{tabular}

It is worth noting there is no guarantee that we can find a set of parameters for which the 0D simulation has exactly the same global outputs values as the $3 \mathrm{D}$ simulation. In fact, we can observe in Table 3 that some coupling outputs do not have the same values in a $3 \mathrm{D}$ sigma-simulation and the 0D corresponding coupled simulations. We will see in a subsequent section how this obstacle can be overcome.

We point out that there are many possibilities to choose the sets of coupling outputs $\mathscr{O}^{\prime}$ and coupled oD parameters. For example, another possibility would have been to use directly the set of outputs to approximate $\mathscr{O}$. This would have lead to $0 \mathrm{D}$ and $3 \mathrm{D}$ simulations with the same stroke volume, but not necessarily the same minimal and maximal volumes. In general the sets of $\mathscr{O}^{\prime}$ and $\mathscr{O}$ have to be related so that it is possible to calculate the values of the outputs $\mathscr{O}$ from the values of the coupling outputs $\mathscr{O}^{\prime}$.

Similarly, there are many possibilities to choose the sets of coupling OD parameters. Here we could also have set the resting radius in the $0 \mathrm{D}$ model to a value for which the "resting volume" is the same than in the $3 \mathrm{D}$ model, then estimate only the stiffness and contractility of the 0D model. Empirically, it seems to be a good thing to use more parameters to avoid ending in a local minimum during the parameter estimation of the $O D$ simulations.

\subsection{Parameter Mapping: A function to Convert 3D model Parameters into 0D model Parameters}

We now have a corresponding coupled $O D$ simulation with parameters $Y_{i} \in \Omega_{0 D}$ for each sigma-simulation with parameters $X_{i} \in \Omega_{3 D}$. The second idea of the coupling is to build a mapping $\phi$ between the $3 \mathrm{D}$ and 0D model parameters using the $X_{i}$ and $Y_{i}$. This mapping will then be used to approximate global outputs 

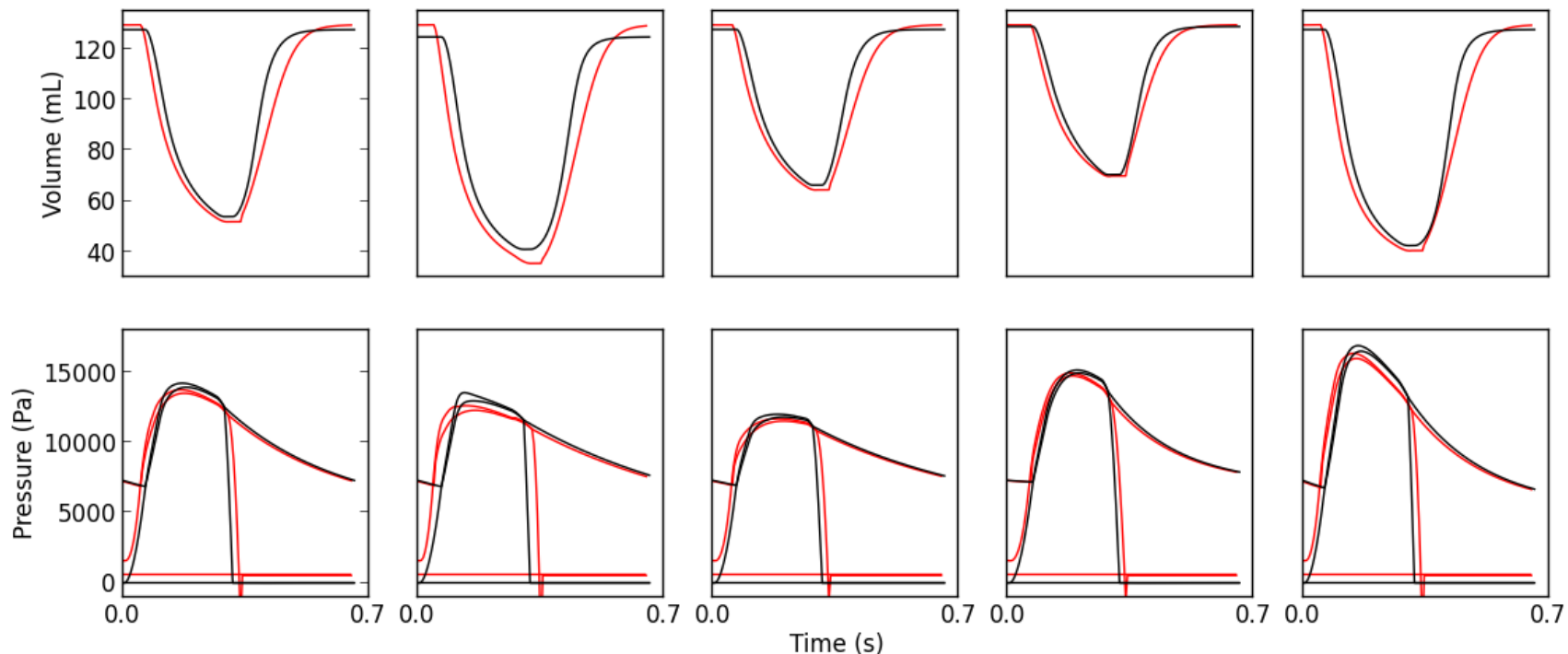

Fig. 3: Comparison between the volume (top) and pressure (bottom) curves of the sigma-simulations simulated with the $3 \mathrm{D}$ model (red), and the corresponding coupled $0 D$ simulations (black). The 5 columns correspond respectively to the sigma-simulations with parameters $X_{0}, X_{1}^{+}, X_{2}^{+}, X_{1}^{-}$and $X_{2}^{-}$.

values $\mathscr{O}_{3 \mathrm{D}}\left(x_{i}\right)$ of the $3 \mathrm{D}$ simulations with parameters $x_{i}$, from the values $\mathscr{O}_{0 \mathrm{D}}\left(\phi\left(x_{i}\right)\right)$ of the $0 \mathrm{D}$ simulations with parameters $\phi\left(x_{i}\right)$.

The parameters $X_{i} \in \Omega_{3 D}$ were chosen in a specific way in Section 3.2: one $\left(X_{0}\right)$ is at the center of the $x_{i}$ and there are two equidistant of $X_{0}\left(X_{k}^{+}\right.$and $X_{k}^{-}$for $\left.k=1 . . N_{2}\right)$ for each of the $N_{2}$ axis, which are orthogonals from each other. However, the $Y_{i}$ were independently estimated for each sigma-simulation so there is no such relationship.

For the mapping $\phi$ we use here a degree 2 hypersurface which interpolates the $Y_{i}$ in the points $X_{i}$. In dimension 1 this is equivalent to finding a degree 2 polynomial which interpolates three specific points. In higher dimension ( $N_{2}$ in our case), there is a straightforward formula because of the specific organisation of $X_{i}$ along orthogonal axis:

$\left\{\begin{array}{l}c_{k}=\left(\mathbf{x}-\mathbf{X}_{\mathbf{0}}\right) \cdot \frac{\mathbf{d}_{\mathbf{k}}}{\left\|\mathbf{d}_{\mathbf{k}}\right\|^{2}}, k=1 . . N_{2} \\ \mathbf{F}_{\mathbf{k}}^{+}=\frac{\left(Y_{k}^{+}-Y_{0}\right)+\left(Y_{k}^{-}-Y_{0}\right)}{2}, k=1 . . N_{2} \\ \mathbf{F}_{\mathbf{k}}^{-}=\frac{\left(Y_{k}^{+}-Y_{0}\right)-\left(Y_{k}^{-}-Y_{0}\right)}{2}, k=1 . . N_{2} \\ \phi(x)=Y_{0}+\sum_{k=1}^{N_{2}} c_{k} \cdot\left(c_{k} \cdot \mathbf{F}_{\mathbf{k}}^{+}+\mathbf{F}_{\mathbf{k}}^{-}\right) .\end{array}\right.$

This formula leads to $\phi\left(X_{i}\right)=Y_{i}$ for all the $i=$ $1 . .2 N_{2}+1$ sigma-simulations, so the parameters of the $3 \mathrm{D}$ sigma-simulations are mapped to the parameters of the coupled $O D$ simulations of the previous section. We will then use this mapping to approximate global outputs of $3 \mathrm{D}$ simulations with parameters $x_{i}$ from $0 \mathrm{D}$ simulations with parameters $\phi\left(x_{i}\right)$.

\subsection{Approximating global outputs: Correcting Bias}

Ideally in the computation of coupled $O D$ simulations in Section 3.3, we find 0D simulations with the same coupled outputs values than the 3D sigma-simulations i.e. $\mathscr{O}^{\prime}{ }_{3 \mathrm{D}}\left(X_{i}\right)=\mathscr{O}^{\prime}{ }_{3 \mathrm{D}}\left(Y_{i}\right)$. As illustrated in Table 3 , this is not always the case and the coupled outputs values can be different between the coupled $O D$ simulations and the sigma-simulations. This also means that the direct approximation of the sigma-simulations output values $\mathscr{O}_{3 \mathrm{D}}\left(X_{i}\right)$ by the values $\mathscr{O}_{0 \mathrm{D}}\left(Y_{i}\right)$ through the mapping has a bias due to this difference.

In order to correct this approximation bias, both for the output values of the sigma-simulations and all the subsequent 3D simulations with parameters $x_{i}$, we build a new degree 2 hypersurface $\psi$ between the parameters of the sigma-simulations $X_{i}$ and the bias. The formula is exactly the same as in Equation 1 where the $Y_{i}$ are replaced by the bias values $\left(\mathscr{O}_{3 \mathrm{D}}\left(X_{i}\right)-\mathscr{O}_{0 \mathrm{D}}\left(Y_{i}\right)\right)$.

The final approximating function $\mathscr{C}_{\phi, \psi}$ used to approximate the $\mathscr{O}_{3 \mathrm{D}}\left(x_{i}\right)$ is then given by the following formula:

$\mathscr{C}_{\phi, \psi}\left(x_{i}\right)=\mathscr{O}_{0 \mathrm{D}}\left(\phi\left(x_{i}\right)\right)+\psi\left(x_{i}\right) \approx \mathscr{O}_{3 \mathrm{D}}\left(x_{i}\right)$,

and interpolates in particular the global outputs values $\mathscr{O}_{3 \mathrm{D}}\left(X_{i}\right)$ of the sigma-simulations.

\subsection{Approximation Results}

Results are given here for the approximation of the global outputs values $\mathscr{O}_{3 \mathrm{D}}\left(x_{i}\right)$ of the 30 simulations with 
parameters $x_{i}$. We compute the mean absolute error made on the approximation of the 3 global outputs $\mathscr{O}$, first with the biased approximation with $\mathscr{O}_{0 \mathrm{D}}\left(\phi\left(x_{i}\right)\right)$ ( $\left.M A E_{\text {Biased }}\right)$, then with the corrected approximation with $\mathscr{C}_{\phi, \psi}\left(M A E_{\text {Corrected }}\right)$. Results are reported in Table 4 .

We observe a good approximation of the output values compared to the range of values to be approximated, and that the corrected approximation makes a better approximation of the outputs values than the biaised approximation. This means the hypersurface $\psi$ indeed corrects errors due to the differences between the coupled $O D$ simulations and the $3 \mathrm{D}$ sigma-simulations.

Table 4: Error in the approximation of the global outputs values $\mathscr{O}_{3 \mathrm{D}}\left(x_{i}\right)$ with the various methods.

\begin{tabular}{l|lll} 
Global Output & $\mathrm{SV}(\mathrm{ml})$ & $\mathrm{DP}(\mathrm{Pa})$ & $\mathrm{MP}(\mathrm{Pa})$ \\
\hline Range & 38.23 & 3010 & 2254 \\
\hline$M A E_{\text {Corrected }}$ & 1.59 & 56.4 & 137 \\
$M A E_{\text {Biased }}$ & 4.58 & 62.8 & 140 \\
$M A E_{\text {Hypersurface }}$ & 2.09 & 511 & 408 \\
\hline$M A E_{\text {Hypersurface-11 }}$ & 0.25 & 174 & 93 \\
\hline
\end{tabular}

Finally, we compare our method to an interpolation with a degree 2 hypersurface ( $\left.M A E_{\text {Hypersurface }}\right)$. To this end we use the same formula than Equation 1, where the $Y_{i}$ are replaced by the output values $\mathscr{O}_{3 \mathrm{D}}\left(X_{i}\right)$. We see in particular that our method performs better on all the outputs ( $M A E_{\text {Corrected }}<M A E_{\text {Hypersurface }}$ ), in particular on the pressure values. This is because the sigma-simulations are computed only in the directions of maximal variations of the parameters $\sigma_{0}$ and $c_{1}$ (see Section 3.2). There is then a few directions of the parameter space in which the variations of global output values could not be evaluated by the interpolation.

In order to compare more fairly to an interpolation method, we computed the sigma-simulations in all the directions of the domain by selecting all the eigenvectors in Section 3.2, leading to $2 \cdot N_{1}+1=11$ sigmasimulations. We performed the degree 2 interpolation from these 11 sigma-simulations and report the results ( $\left.M A E_{\text {Hypersurface-11 }}\right)$. The degree 2 hypersurface performs better than our method on the stroke volume and the mean pressure but not on the diastolic pressure.

We conclude that the approximation using the coupling of the $0 \mathrm{D}$ and $3 \mathrm{D}$ models gives competitive approximation results compared to the classical regression methods, and with the lowest computational cost. This is because the variations of some outputs (which rely on the same equations in both models) can be directly approximated in some directions of the parameter space, without having to compute 3D simulations in these directions. Here in particular, the pressure outputs variations due to changes in the haemodynamic parameters $C, R_{p}$ and $P_{\text {ve }}$ are correctly predicted with the coupling (especially the Diastolic Aortic Pressure (DP) variations), even though no sigma-simulation was computed in the directions of maximal variation of these parameters (Section 3.2). As a consequence, only 5 sigmasimulations are required to approximate all the outputs values within the parameter space with the coupling, while the hypersurface interpolation needs 11 sigmasimulations to achieve similarly accurate results.

\section{Multi-Fidelity Optimization for Efficient 3D Cardiac Model Personalisation}

Here we present our multifidelity personalisation method for the 3D model. We suppose a parameter estimation with CMA-ES was set up over $N_{1}$ parameters $\mathscr{P}$ of the 3D model as described in Section 2.3, some global outputs $\mathscr{O}$, some target values $\widehat{\mathscr{O}}$ and a population size $m$. The idea of the method is to replace the scores of 3D simulations in CMA-ES with approximate scores calculated through multifidelity coupling.

We illustrate the method with the same set of 5 parameters $\mathscr{P}$ and 3 outputs $\mathscr{O}$ as in Section 3 and the same number $m=30$ for the population size. Target values $\widehat{\mathscr{O}}$ for the optimization are respectively $60 \mathbf{~ m l}$ for the Stroke Volume (SV), 7315 Pa for the Diastolic Aortic Pressure (DP) and 10152 Pa for the Mean Aortic Pressure (MP). The normalisation coefficients for this problem (in the vector $\mathscr{N}$ defined in Section 2.3.1) are $10 \mathbf{~ m l}$ for the Stroke Volume (SV), $200 \mathbf{P a}$ for the Diastolic Aortic Pressure (DP) and the Mean Aortic Pressure (MP).

\subsection{Multi-fidelity-CMA: CMA-ES Optimisation with the Multifidelity Coupling}

At each iteration, the algorithm CMA-ES asks for the scores of $m$ simulations of the 3D model, whose parameters $x_{j}$ are drawn from a multivariate distribution.

A first approach to replace the computation of the $3 \mathrm{D}$ simulations by $\mathrm{OD}$ simulations is to perform the coupling described in Section 3 for each generation of CMA-ES. This means recomputing sigma-simulations, coupled $O D$ simulations and a parameter mapping for each set of $x_{j}$. This was our approach (called Coupled$C M A$ ) in (Mollero et al., 2016). We showed that the optimisation could converge with approximate scores, even as fast as with the real scores in some cases. We 


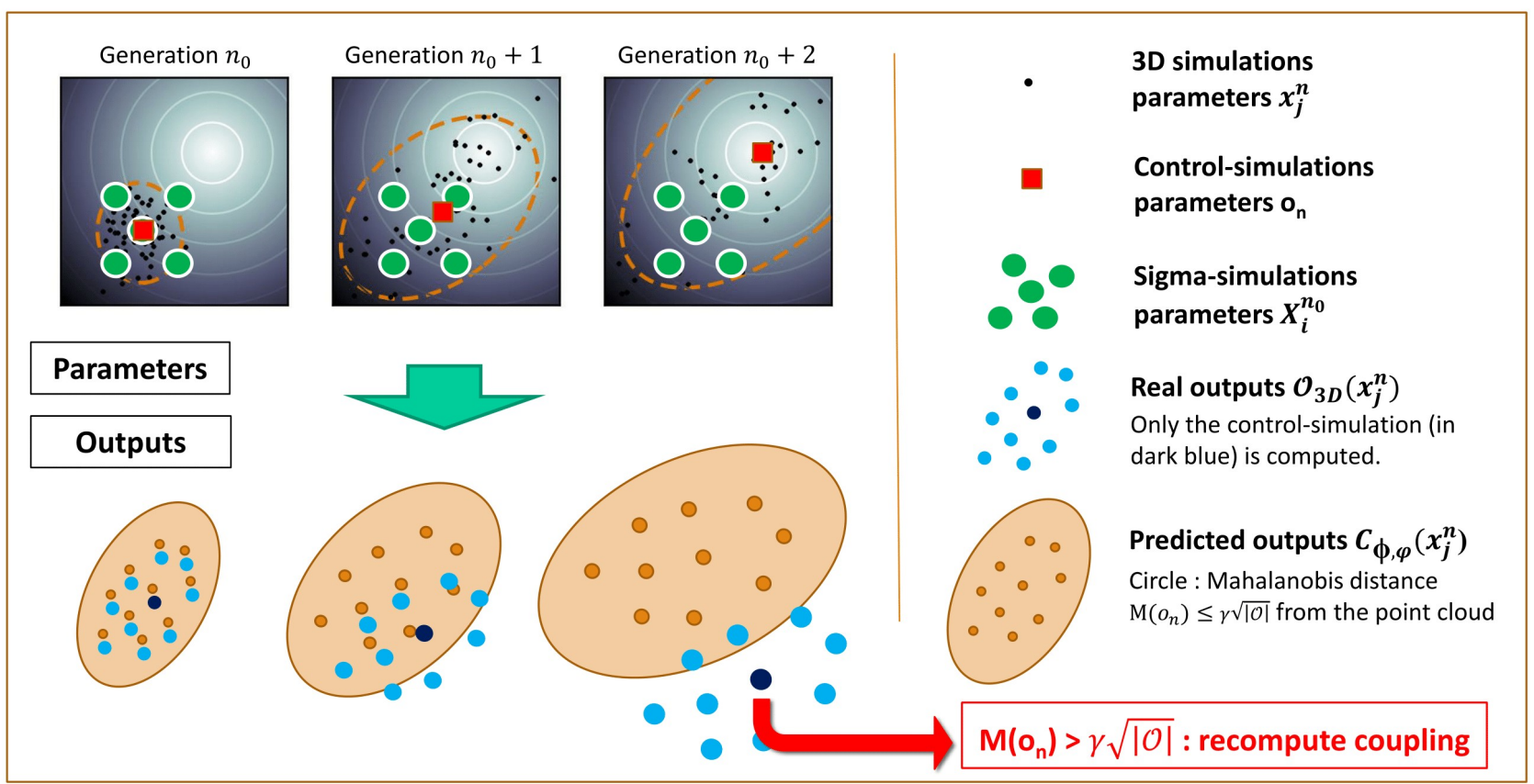

Fig. 4: Criteria for selecting the generation for the next coupling step in the selection step: as the 3D parameters of the simulations asked by CMA-ES (in black) are increasingly far from the sigma-simulations (in green) of the coupling, the predicted outputs values with 0D simulations (in orange) are increasingly far from the real outputs values of the 3D simulations. We then recompute the coupling when this distance is too high $\left(M\left(o_{n}\right)>\gamma \sqrt{|\mathscr{O}|}\right)$.

also personalised 34 hearts with this method, thus exhibiting a practical personalisation method with a lower computational burden than the original CMA-ES algorithm (because only the sigma-simulations were computed for each generation instead of the $m$ 3D simulations).

Here we present an improved approach called Multifidelity-CMA. Instead of recomputing the coupling for each generation, we approximate scores of $3 \mathrm{D}$ simulations of successive generations of CMA-ES. Indeed, because the sets of parameters $x_{j}^{n}$ and $x_{j}^{n+1}$ asked by CMA-ES in two consecutive generations $n$ and $n+1$ are usually close, the function $\mathscr{C}_{\phi, \psi}$ computed at the iteration $n$ to approximate $3 \mathrm{D}$ simulations with parameters $x_{j}^{n}$, can give a good approximation for $3 \mathrm{D}$ simulations with parameters $x_{j}^{n+1}$ as well.

On the other hand, after a few iterations $n+1 . . n+p$, the points asked by CMA-ES can be increasingly far from the sigma-simulations of the multifidelity coupling performed at $n$. This can lead to approximations of the scores which are increasingly inaccurate, making the optimisation impossible.

We then developed a criterion to evaluate the accuracy of the approximation for a few successive iteration of CMA-ES, then decide at which step a new multi- fidelity coupling has to be computed. This is done by iterating on the following steps:

1. Coupling step. At a generation $n_{0}$ of CMA-ES, we first perform a multifidelity coupling, as explained in 3.4. This leads to the computation of the function $\mathscr{C}_{\phi, \psi}$.

2. Exploration step. Then, we perform $N$ iterations $n=n_{0}+1 . . n_{0}+N$ of the CMA-ES algorithm, where all the outputs $\mathscr{O}_{3 \mathrm{D}}\left(x_{j}^{n}\right)$ of the $3 \mathrm{D}$ simulations with parameters $x_{i}^{n}$ are approximated by $\mathscr{C}_{\phi, \psi}\left(x_{j}^{n}\right)$.

3. Control step. For each of these $N$ iterations, we compute a control-simulation: the 3D simulation whose parameters $o_{n}$ are the mean of the population parameters $x_{j}^{n}$.

4. Selection step. We compute our criterion $M\left(o_{n}\right)$ as the Mahalanobis distance between the vector of outputs values $\mathscr{O}_{3 \mathrm{D}}\left(o_{n}\right)$ of the control-simulation and the set of vectors of approximated outputs values $\mathscr{C}_{\phi, \psi}\left(x_{j}^{n}\right)$.

Finally we select the iteration $n *$ at which the next coupling step is performed with the following criteria:

$$
n *=\underset{M\left(o_{n}\right)<\gamma \sqrt{|\mathscr{O}|}}{\operatorname{argmin}} \mathscr{O}_{3 \mathrm{D}}\left(o_{n}\right)
$$

The process is illustrated in Fig 4. The Mahalanobis distance $M\left(o_{n}\right)$ is a ratio between the approximation 
error on the control-simulation output values, and the range of approximate outputs values for this generation. Roughly, this gives an indication on "how accurate the coupling is" on the control-simulation, compared to "how accurate it needs to be" so that CMA-ES can rank the scores accurately.

For example in Fig 5, we report for $N=10$ iterations the scores of the control-simulations $o_{n}$ which were predicted through the function $\mathscr{C}_{\phi, \psi}$ (in black), and the real scores of these simulations (in blue). Simultaneously, we show the criterion $M\left(o_{n}\right)$ for these $N$ iterations and the upper value (red line) $\gamma \sqrt{|\mathscr{O}|}$ for the criterion $(\gamma=1.5$ here $)$.

We can see that the score prediction (thus the approximation of the outputs $\mathscr{O}_{3 \mathrm{D}}\left(x_{j}^{n}\right)$ values by $\left.\mathscr{C}_{\phi, \psi}\right)$ is quite accurate for at least the 5 first iterations, and is less accurate for $n \geq 6$. Then, even though the score prediction seems as accurate at the iteration 5 than at the generation $1, M\left(o_{n}\right)$ is higher. This is because the prediction error is more important relatively to the set of $\mathscr{C}_{\phi, \psi}\left(x_{j}^{n}\right)$ of the generation, in particular in directions where the set has a lower variance.

In this example, the iteration 5 was selected to recompute the coupling (black vertical line), which is also the iteration where the control simulation has the minimal score over the 10 iterations. In some cases, later iterations can have a lower score but are not selected because the criterion $M\left(o_{n}\right)$ is too high for this iteration (such as the iteration 7 ).

The upper bound $\gamma \sqrt{|\mathscr{O}|}$ for the criterion has an important impact on the optimisation behavior. If a high accuracy is imposed (small $\gamma$ value), then one of the earlier iterations of the exploration step is usually selected for the subsequent coupling step, even if a later control-simulation has a lower score. This can lead to a slow optimization. On the other with a small accuracy (high $\gamma$ value) the CMA-ES algorithm can end up in local minima because it performed the optimization on inaccurate values.

Therefore the value of $\gamma$ characterizes a trade-off between maximising the optimisation gain with a single coupling, and ensuring the approximation errors do not impact the optimisation process. Because of the probabilistic nature of the algorithm and the various non-linearities of the score function, the optimal value of $\gamma$ seems very dependent on the optimisation problem. We found $\gamma=1.5$ to give good convergence results in our experiments and the number $n *$ of the iteration selected in the selection step is 5.5 in average in our experiments.
Fig. 5: Top: real scores (blue) and approximated scores (black) of the $\mathrm{N}=10$ control-simulations. Bottom: Value of the criterion $M\left(o_{n}\right)$ of the control-simulations.
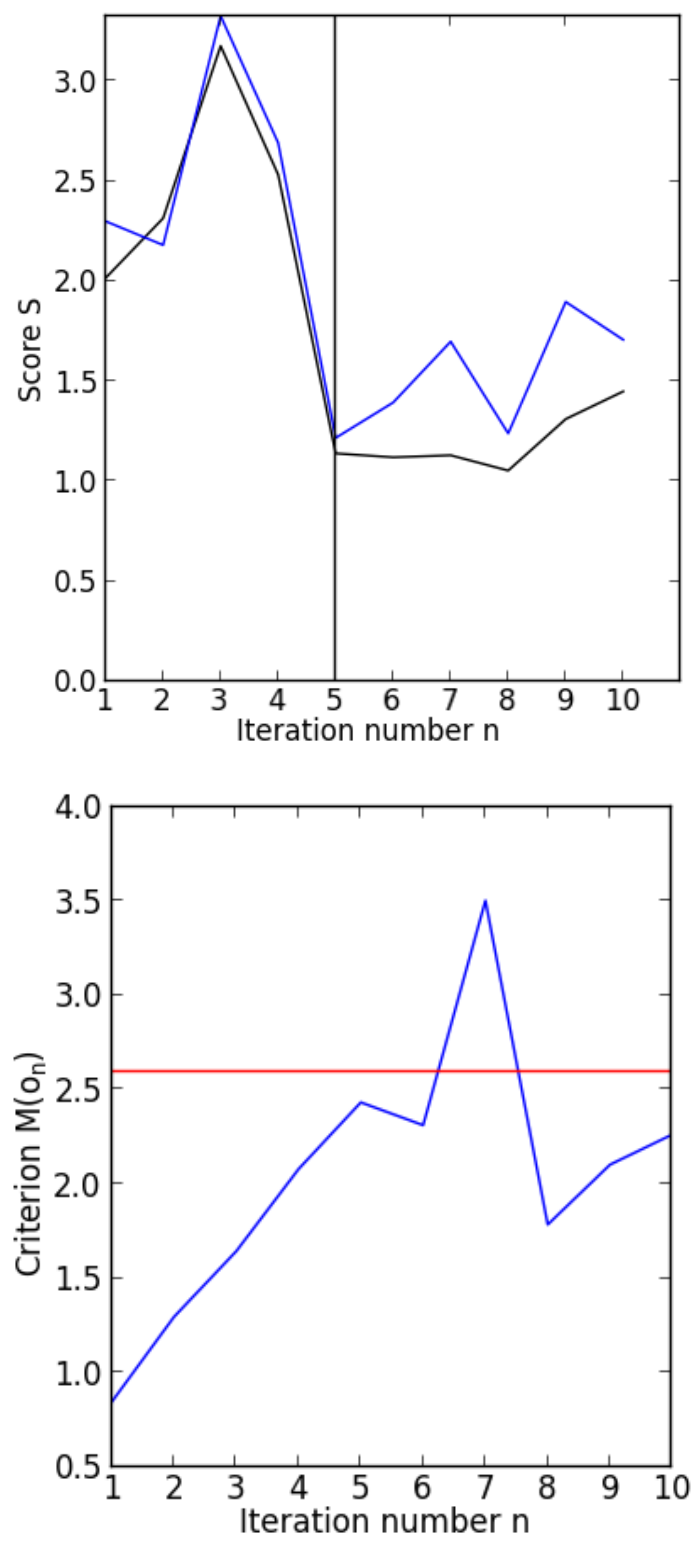

4.2 Computational Considerations: A Parallelizable Method

The main computational cost in personalisation methods comes from the computation of the 3D simulations. In our implementation, each simulation of one heartbeat with the 3D model uses one CPU, during a time $T_{3 D}$ which depends mostly on the size of the mesh, and the heartbeat duration.

Most of the modern research is performed on computer clusters which can perform many tasks at the same time. In particular in our method, many steps 
can be parallelized. To compare different optimization methods in a parallel setting, we introduce here two metrics: the classic CPU Time which measures the total amount of CPU resources used, and the Optimization Time which measures the duration of the optimization in (real) time.

During one complete iteration of Multifidelity-CMA, the following steps are parallelized:

1. Computation of the $2 N_{2}+13 \mathrm{D}$ sigma-simulations: the simulations are performed in parallel and each one takes a CPU Time $T_{3 D}$. The whole step has then a $C P U$ Time of $\left(2 N_{2}+1\right) \cdot T_{3 D}$ and an Optimization Time of $T_{3 D}$

2. Computation of the coupled OD simulations: all the parameter estimations are performed in parallel. Each one uses 4 CPUs during fixed time of around 3 minutes. The whole step has a CPU Time of $\left(2 N_{2}+1\right) \cdot 4 \cdot 3$ minutes and an Optimization Time of 3 minutes.

3. Computation of the $N 3 \mathrm{D}$ control-simulations: the simulations are performed in parallel and each one takes a CPU Time $T_{3 D}$. The whole step has a $C P U$ Time of $N \cdot T_{3 D}$ and an Optimization Time of $T_{3 D}$

In our example we have 5 sigma-simulation and 10 control-simulation, and the 3D simulation takes 15 minutes. Each iteration of Multifidelity-CMA then takes a total CPU Time of $5^{*} 15+4^{*} 5^{*} 3+10^{*} 15=285$ minutes and an Optimization Time of 33 minutes.

4.3 Results: Comparison of Optimization Time, CPU Time for 4 personalizations methods

Here we compare the evolution of the CPU Time and the score $\mathrm{S}$ during optimization on a typical case, with the 4 following optimization methods:

1. The Multifidelity-CMA method with $0 \mathrm{D} / 3 \mathrm{D}$ coupling.

2. The Multifidelity-CMA method where the approximation of outputs is done with a degree 2 hypersurface interpolation relying on 11 sigma-simulations (as explained in Section 3.6).

3. The classic CMA-ES method with a population size of $m=30$.

4. BOBYQA, which is another commonly used gradient-free optimizer for example to solve personalisation problems (Seegerer et al., 2015) or as a baseline to evaluate other personalisation methods (Neumann et al., 2016). It uses trust region method and forms successive quadratic models of the score function which interpolates the points computed during optimization.
Fig. 6: Comparison of the evolution of the score S (top) and CPU Time (bottom) during optimization for the four methods. BOBYQA is in red, the classic CMAES is in blue, Multifidelity-CMA with the hypersurface approximation is in black and Multifidelity-CMA with $0 \mathrm{D} / 3 \mathrm{D}$ coupling is in green.
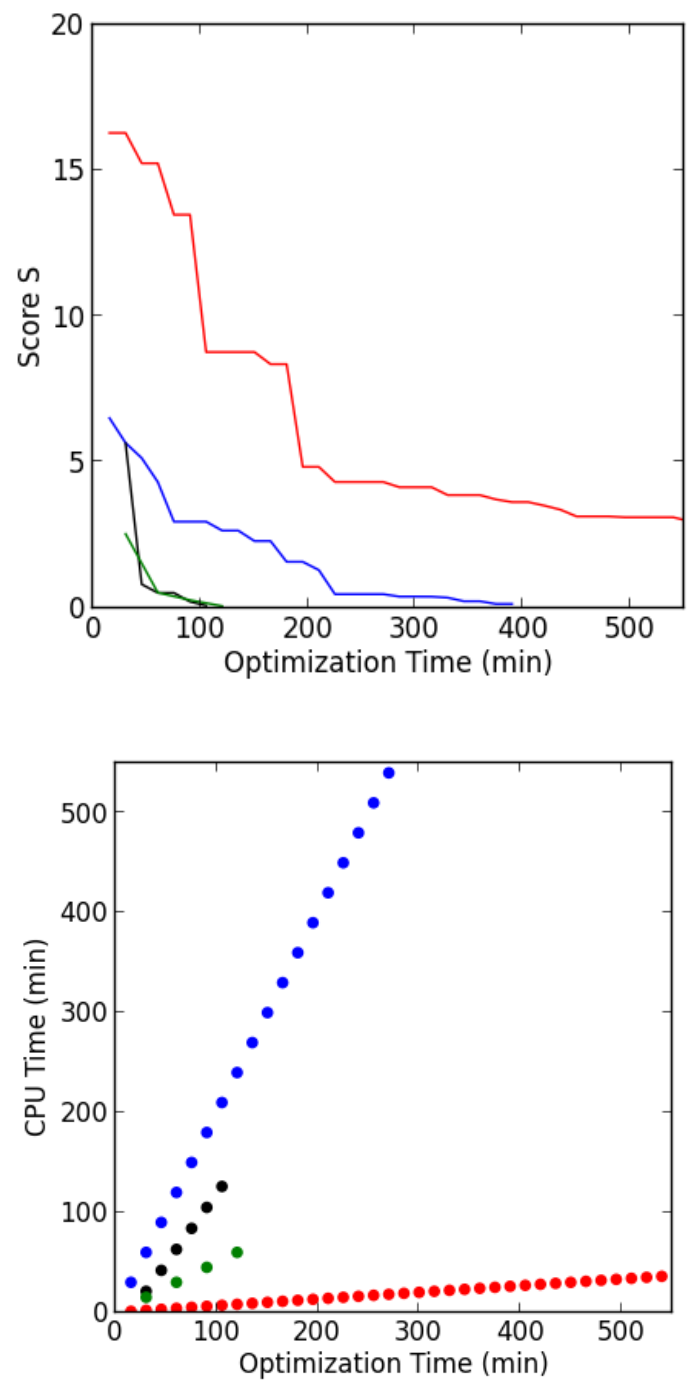

Results are shown in Figure 6. We can see that BOBYQA (red) is slow to converge, but has also a low computational cost, both due to the fact that BOBYQA performs only one iteration at a time. The normal CMA-ES (blue) converges faster than BOBYQA, but with a very high computational cost because 30 simulations of the 3D model are computed at each generation.

Finally, both our multifidelity approaches are very fast to converge, however the Multifidelity-CMA which uses the $0 \mathrm{D} / 3 \mathrm{D}$ multifidelity coupling is the one with the lowest CPU Time (because only 5 sigma- 
simulations per complete iteration is computed instead of 11, as explained in Section 3.6).

We conclude than the multifidelity approach of the CMA-ES algorithm leads to considerable improvements in optimization speed, both from the original CMA-ES algorithm and BOBYQA. Finally, the approximation of outputs with a $0 \mathrm{D} / 3 \mathrm{D}$ multifidelity coupling instead of a generic hypersurface interpolation leads to additional computational gains.

\subsection{Results: Personalisation of a database of 121 cases}

We finally present results on a large database of 121 cases. For each patient, a biventricular heart mesh geometry (between 10000 and 15000 nodes) was built from the available MRI image and the boundaries of the myocardium were tracked in the cine MRI images as described in (Jolly et al., 2011) and (Wang et al., 2013). This led to the computation of the volume curve, then the value of the stroke volume. Pressure measurements were also available for each heartbeat.

We applied our Multifidelity-CMA method to personalise the whole cohort. The optimization started from a vector $x_{\text {start }}$ of parameter values which has the same values for every patient, except for $P_{v e}$, which is set at the value $D P-2000 P a$ (see Table 6 ). The algorithms ran for around 2.5 days, and the BOBYQA optimization was ran on the same problems during this period as well.

We consider a personalisation to be successful when a set of parameter values was found with a score lower than $l_{1}=0.1$, and acceptable if the score is lower than $l_{2}=1$. This means the personalised simulation matches the target stroke volume within $1 \mathrm{ml}$ and the pressure measurements within $20 \mathrm{~Pa}$ for the successful case, and within respectively $10 \mathrm{ml}$ and $200 \mathrm{~Pa}$ in the acceptable case. In other cases the personalisation is said failed. We report the number of successful, acceptable and failed cases on this database, for both methods in Table 5 .

Table 5: Results of the personalisation on the database.

\begin{tabular}{l|lll} 
Result & Successful & Acceptable & Failed \\
\hline Multifidelity-CMA & 113 & 6 & 2 \\
BOBYQA & 5 & 69 & 47 \\
\hline
\end{tabular}

A high number of cases were successfully personalised (113 among 121 cases) with our method. For the acceptable cases, and one of the failed case, the optimization had converged in a local minima. For the other failed case, the CMA-ES algorithm diverged to extreme parameter values during optimization. For BOBYQA, the convergence was not yet reached in most of the non-successful cases (the score is the lowest in the last iteration).

We finally report the mean and standard deviation of all the estimated parameter values, in Table 6, as well as the norm of their relative variation $|\Delta|$ compared to the starting value during the optimization. This shows in particular that the stiffness $c_{1}$ did not change a lot during the personalisation process. The arterial compliance $C$ and the contractility $\sigma_{0}$ are the parameters which changed the most.

\section{Discussion and Conclusion}

We presented a novel multifidelity approach involving a 3D cardiac electromechanical cardiac model and a simplified 0D model, which relies on the same equations but with simplifying assumptions. We developed an original multifidelity coupling between the parameters of both models, which gives a good multifidelity approximation of global output values in 3D simulations from $0 \mathrm{D}$ simulations. We then used this approximation in an efficient parameter estimation process using the genetic algorithm CMA-ES, in order to have an efficient multifidelity personalisation method for the 3D model.

Our multifidelity coupling procedure computes a mapping between the parameters of a few representative 3D sigma-simulations within the domain, and the parameters of corresponding coupled $O D$ simulations with the same output values. This is done through parameter estimation on the $0 \mathrm{D}$ model parameters to compute coupled $O D$ simulations that have the same global outputs values than 3D sigma-simulations. The parameter mapping is then derived through an interpolation method.

This enables to get fast and accurate approximations of 3D simulations with the 0D model. These approximations are then used in the parameter estimation of 3D model parameters with CMA-ES, to replace 3D simulations while simultaneously controlling the accuracy of the approximation and recomputing a coupling when the accuracy is too low. Ultimately, this results into both an increase of the speed of the 3D parameter estimation process and a decrease of the computational cost.

Our multifidelity approach slightly differs from more classic multifidelity methods (Kennedy and O'Hagan, 2000; Peherstorfer et al., 2016) where the same parameter values are used as input of both models, and the outputs of the low-fidelity model are corrected a posteriori to fit the outputs of the high-fidelity model. Since 
Table 6: Statistics of the estimated parameter values and their variations during the personalisation.

\begin{tabular}{l|ccccc} 
& $c_{1}(\mathrm{kPa})$ & $\sigma(\mathrm{MPa})$ & $P_{\text {ve }}(\mathrm{Pa})$ & $R\left(\mathrm{MPa} \cdot \mathrm{m}^{3} \cdot \mathrm{s}\right)$ & $\mathrm{C}\left(\mathrm{MPa}^{-1} \cdot \mathrm{m}^{-3}\right)$ \\
\hline$x_{\text {start }}$ & $50.1 \mathrm{e}^{1}$ & 68.8 & $\mathrm{DP}-2000$ & 54.1 & $18.0 \mathrm{e}^{-3}$ \\
\hline Mean & $50.5 \mathrm{e}^{1}$ & 91.6 & 4760 & 68.2 & $8.17 \mathrm{e}^{-3}$ \\
Std. & $0.36 \mathrm{e}^{1}$ & 36.5 & 1340 & 16.3 & $2.41 \mathrm{e}^{-3}$ \\
\hline Mean $|\Delta|$ & $4.74 \%$ & $53.9 \%$ & $26.2 \%$ & $35.8 \%$ & $54.7 \%$ \\
\hline
\end{tabular}

the parameters of both models are not exactly the same, we had to find a mapping between the parameters instead of the outputs. This was tractable thanks to the fast parameter estimation in the 0D model.

A first extension of the multifidelity coupling would be to use additional shared parameters and equations in both models, to approximate a larger variety of outputs of the 3D model (e.g. flow velocities, timings of valve opening and closing). Since CMA-ES has already been proven successful on complex optimisation problems with a larger parameter space, we expect the personalisation method to scale well. A second extension would be to use the multifidelity personalisation to personalise "geometrical" or "local" measurements which are outputs of the 3D model but not of the 0D model (e.g. the septal shortening or the circumferential torsion). Indeed, even though they cannot be approximated through the 0D/3D multifidelity coupling, their values can still be locally approximated during personalisation using the hypersurface interpolation.

Finally, the lower-fidelity approximation could be used not only for personalisation but also for other applications that require many simulations, such as parameter sensitivity or uncertainty quantification (with Monte-Carlo methods for example) and also for applications simulations that require the computation of many cardiac cycles. In particular, a case where the multifidelity approach could be useful is when the 3D model is coupled with a full-body circulation model as boundary conditions. Indeed, studies associated to such models (for example on the influence of physical exercise, increased heart rate and/or pressure loads) usually require many heartbeats to be computed. This can be computationally intensive with the $3 \mathrm{D}$ model but, it could be done faster using 0D simulations, through a similar coupling method than in this manuscript. In this case where the number of coupled parameters would be high, additional constraints could be added in the parameter mapping to impose correlations between parameters with different equations or values but a similar behavior.

Compliance with Ethical Standards : Informed consent was obtained from the subjects and the protocol was approved by the local Research Ethics Committee.

Funding: This work has been partially funded by the European Union FP7-funded project MD-Paedigree (Grant Agreement 600932) and contributes to the objectives of the European Research Council advanced grant MedYMA (2011-291080).

Conflict of Interest: The authors declare that they have no conflict of interest.

\section{APPENDIX A: Mechanical Equations and Haemodynamics}

As described in (Marchesseau et al., 2013a) our 3D electromechanical model is based on the Bestel-Clement-Sorine model (BCS) of sarcomere contraction as extended by Chapelle et al. (2012), in conjunction with a Mooney-Rivlin energy for the passive hyperelasticity. Hemodynamics are represented through global values of pressures and flows in the cardiac chambers, and coupled to the mechanical equations with the Windkessel model of blood pressure for the after-load (aortic pressure).

\subsection{The BCS model: Active Contraction and Passive Material}

The BCS model describes the sarcomere forces as the sum of an active contraction force in the direction of the fibre, in parallel with a passive isotropic visco-hyperelastic component (see Fig 1.b). It is compatible with the laws of thermodynamics, and allows to model physiological phenomena at the sarcomere scale which translate at the macroscopic scale (such as the Starling Effect).

The active force in the sarcomere is modeled by the filament model of Huxley (1957), which describes the binding/unbinding process of the actin and myosin in the sarcomere at the nanoscopic scale. At the mesoscopic scale, it results (Caruel et al., 2014) in a differential equation which relates the active stress $\tau_{\mathrm{c}}$, the stiffness $k_{\mathrm{c}}$ and the strain $e_{\mathrm{c}}$ of the filament within the sarcomere:

$\left\{\dot{k_{\mathrm{c}}}=-\left(|u|_{+}+|u|_{-}+\alpha\left|\dot{e_{\mathrm{c}}}\right|\right) k_{\mathrm{c}}+k_{0}|u|_{+}\right.$,

$\left\{\dot{\tau_{\mathrm{c}}}=-\left(|u|_{+}+|u|_{-}+\alpha\left|\dot{e_{\mathrm{c}}}\right|\right) \tau_{\mathrm{c}}+\dot{e_{\mathrm{c}}} k_{\mathrm{c}}+\sigma_{0}|u|_{+}\right.$,

where $\alpha$ is a constant related to the cross-bridge destruction during contraction, $k_{0}$ and $\sigma_{0}$ are respectively the maximum stiffness and contraction. The values of $|u|_{+}$and $|u|_{-}$are respectively the rate of build-up $k_{\mathrm{ATP}}$ and decrease $k_{\mathrm{RS}}$ of the force during contraction and relaxation, which depends on 
the depolarisation and repolarisation times $T_{d}$ and $T_{r}$ of the sarcomere:

$$
u= \begin{cases}k_{\mathrm{ATP}} & \text { when } T_{d} \leq t \leq T_{r} \\ -k_{\mathrm{RS}} & \text { otherwise } \\ |u|_{+}=\max (u, 0), & \\ |u|_{-}=-\min (u, 0) & \end{cases}
$$

This active force is applied in the direction of the fibre through the visco-elastic component, made of a spring $E_{s}$ and a dissipative term $\mu$ (see Fig 1.b). As derived in Caruel et al. (2014), the resulting stress $\sigma_{1 \mathrm{D}}$ in the fibre direction is given by:

$$
\left\{\begin{array}{l}
\sigma_{1 \mathrm{D}}=E_{s} \frac{e_{1 \mathrm{D}}-e_{\mathrm{c}}}{\left(1+2 e_{\mathrm{c}}\right)^{2}}, \\
\left(\tau_{c}+\mu \dot{e_{c}}\right)=E_{s} \frac{\left(e_{1 \mathrm{D}}-e_{\mathrm{c}}\right)\left(1+2 e_{1 \mathrm{D}}\right)}{\left(1+2 e_{\mathrm{c}}\right)^{3}},
\end{array}\right.
$$

where $e_{1 \mathrm{D}}=\underline{\tau_{1}} \cdot \underline{\underline{e}} \cdot \underline{\tau_{1}}$ is the strain in the fibre direction $\tau_{1}$ ( $\underline{\underline{e}}$ is the Green-Lagrange strain tensor).

Finally for the passive component the isotropic Mooney Rivlin model of hyperelastic material is used, driven by the following strain energy:

$W_{e}=c_{1}\left(I_{1}-3\right)+c_{2}\left(I_{2}-3\right)+\frac{K}{2}(J-1)^{2}$,

where $I_{1}, I_{2}$ and $J$ are the invariants of the Cauchy-Green deformation tensor, $c_{1}, c_{2}$ and $K$ are the parameters of the material.

\subsection{Haemodynamic Model}

To model the influence of blood dynamics during the cardiac circle, the mechanical equations are coupled with a basic circulation model implementing the 4 phases of the cardiac cycle. For a given ventricle, if we note $P_{\text {at }}$ the pressure in the atrium, $P_{\text {ar }}$ the pressure in the artery and $P_{\mathrm{V}}$ the pressure in the ventricle, the phases are the following:

- Diastolic Filling: when $P_{\mathrm{V}} \leq P_{\mathrm{at}}$, the atrial valve is open and the ventricle fills up with blood.

- Isovolumetric contraction: when contraction starts, $P_{\mathrm{V}}$ rises. $P_{\text {at }} \leq P_{\mathrm{V}} \leq P_{\mathrm{ar}}$ and all the valves are closed.

- Systolic Ejection: when $P_{\mathrm{V}} \geq P_{\mathrm{ar}}$, the arterial valve opens and the blood is ejected into the artery.

- Isovolumetric relaxation: when the contractile forces disappear, $P_{\mathrm{V}}$ finally decreases. $P_{\text {at }} \leq P_{\mathrm{V}} \leq P_{\text {ar }}$ again and all the valves are closed.

We use the haemodynamic model introduced by Chapelle et al. (2012) which links the blood flow $q$ to the ventricular, atrial and arterial pressures with the following equations:

$q= \begin{cases}K_{\mathrm{at}}\left(P_{\mathrm{V}}-P_{\mathrm{at}}\right) & \text { for } P_{\mathrm{V}} \leq P_{\mathrm{at}} \\ K_{\mathrm{iso}}\left(P_{\mathrm{V}}-P_{\mathrm{at}}\right) & \text { for } P_{\mathrm{at}} \leq P_{\mathrm{V}} \leq P_{\mathrm{ar}} \\ K_{\mathrm{ar}}\left(P_{\mathrm{V}}-P_{\mathrm{at}}\right)+K_{\mathrm{iso}}\left(P_{\mathrm{ar}}-P_{\mathrm{at}}\right) & \text { for } P_{\mathrm{V}} \geq P_{\mathrm{ar}}\end{cases}$

Here the atrial pressure $P_{\text {at }}(t)$ (cardiac preload) is imposed at a constant value $P_{\text {at_lower }}$ except for a pressure bump up to $P_{\text {at_upper }}$ at the beginning of cardiac cycle, to account for the contraction of the atrium before the ventricular contraction. Finally the pressure of the artery $P_{\text {ar }}$ (cardiac afterload) is modeled with the 3-parameters Windkessel model (Westerhof et al., 1969) and coupled to the ventricular outflow $q$ through the equation:

$R_{p} C \dot{P_{\text {ar }}}+P_{\text {ar }}-P_{\mathrm{ve}}=\left(R_{p}+Z_{c}\right) q+R_{p} Z_{c} C \dot{q}$,

where $R_{p}$ is the Peripheral resistance, $Z_{c}$ is the Characteristic impedance, $C$ is the Arterial compliance and $P_{V e}$ is the Central Venous Pressure.

\subsubsection{Implementation}

The passive Mooney Rivlin energy is discretised on the 3D mesh with the MJED (Multiplicative Jacobian Energy Decomposition) method described in (Marchesseau et al., 2010), and the BCS fibre stress and stiffness are computed at each node, separately from the positions and velocities. This allows a fast assembly and a good conditioning of the system of mechanical equations. A Rayleigh damping is then added to account for the viscous global dissipation and finally, the ventricular pressure is computed using a prediction-correction approach, performed after solving the first system of mechanical equations. This efficient algorithm and all the details of the mechanical equations and their 3D discretizations are fully discussed in (Marchesseau et al., 2013a).

\section{APPENDIX B: Reduced Equations of the 0D model}

\subsubsection{Mechanical Equations}

The list of simplified equations of our 0D model is reported in Table 7. Equations (a), (b), (c) and (f) are the same sarcomere and visco-elastic equations than Equations $3 \& 5$, which are calculated once for the whole sphere. $C$ in equations (d), (e), (g) and (h) denotes a component of the simplified CauchyGreen deformation tensor which depends only on $y=R-R_{0}$. $\sigma_{\text {passive }}$ in equation $(\mathrm{g})$ is the stress due to the passive law and $\sigma_{\text {viscosity }}$ in equation $(\mathrm{h})$ is the stress due to an additional viscous damping $\eta$, both expressed as a simple function of $C$ (see (Caruel et al., 2014) for the full derivations). In equation (i), $\Sigma_{\mathrm{sph}}$ is the sum of all the stresses applied to the sphere. Equation ( $\mathrm{j}$ ) is the resulting equation of motion which, coupled with the haemodynamic model $(\mathrm{k})$ and the windkessel equation (l), gives the full system of 3 equations to be solved at each iteration.

\subsubsection{Electrophysiology Equations}

Assuming synchronous and homogeneous electrical activation (and thus sarcomere force) means that all of the ventricle is depolarised simultaneously. This leads to a rate of ventricular pressure rise during the isovolumetric contraction (resp. isovolumetric relaxation) which is very close to the rate of build-up $k_{\mathrm{ATP}}$ (resp. decrease $k_{\mathrm{RS}}$ ) of the active stress $\tau_{c}$. However in $3 \mathrm{D}$, this rate is also very dependent on the time for the ventricle to be fully depolarised, which is roughly the QRS duration.

In order to correct this discrepancy between the models, we adapted the electrical parameter $u$ to take into account the QRS duration. We model the fraction $f_{\text {depo }}$ of the ventricle which is currently depolarised as a piecewise linear function of time which depends on $T_{\mathrm{d} \text {,global }}, T_{\mathrm{r}, \text { global }}$ and $Q R S_{\text {duration }}$. Then the values of $|u|_{+}$and $|u|_{-}$in Equation (a) are adapted to depend on the value of $f_{\text {depo }}$ as described in Table 8 . 


$$
\begin{aligned}
& \left(\dot{k_{\mathrm{c}}}=-\left(|u|_{+}+|u|_{-}+\alpha\left|\dot{e_{\mathrm{c}}}\right|\right) k_{\mathrm{c}}+k_{0}|u|_{+}\right. \\
& \dot{\tau_{\mathrm{c}}}=-\left(|u|_{+}+|u|_{-}+\alpha\left|\dot{e_{\mathrm{c}}}\right|\right) \tau_{\mathrm{c}}+\dot{e_{\mathrm{c}}} k_{\mathrm{c}}+\sigma_{0}|u|_{+} \\
& \left(\tau_{c}+\mu \dot{e}_{c}\right)=E_{s} \frac{\left(e_{1 \mathrm{D}}-e_{\mathrm{c}}\right)\left(1+2 e_{1 \mathrm{D}}\right)}{\left(1+2 e_{\mathrm{c}}\right)^{3}} \\
& C=\left(1+\frac{y}{R_{0}}\right)^{2} \\
& e_{1 \mathrm{D}}=\frac{C-1}{2} \\
& \sigma_{1 \mathrm{D}}=E_{s} \frac{e_{1 \mathrm{D}}-e_{\mathrm{c}}}{\left(1+2 e_{\mathrm{c}}\right)^{2}} \\
& \sigma_{\text {passive }}=4\left(1-C^{-3}\right)\left(c_{1}+c_{2} C\right) \\
& \sigma_{\text {viscosity }}=4 \eta\left(1+C^{-6}\right) \dot{C} \\
& \Sigma_{\mathrm{sph}}=\sigma_{1 \mathrm{D}}+\sigma_{\text {passive }}+\sigma_{\text {viscosity }} \\
& \rho d_{0} \ddot{y}=P_{v}\left(1+\frac{y}{R_{0}}\right)^{2}-\frac{d_{0}}{R_{0}}\left(1+\frac{y}{R_{0}}\right) \Sigma_{\mathrm{sph}} \\
& q=4 \pi R_{0}^{2}\left(1+\frac{y}{R_{0}}\right)^{2} \dot{y}= \begin{cases}K_{\mathrm{at}}\left(P_{\mathrm{V}}-P_{\mathrm{at}}\right) & \text { for } P_{\mathrm{V}} \leq P_{\mathrm{at}} \\
K_{\mathrm{iso}}\left(P_{\mathrm{V}}-P_{\mathrm{at}}\right) & \text { for } P_{\mathrm{at}} \leq P_{\mathrm{V}} \leq P_{\mathrm{ar}} \\
K_{\mathrm{ar}}\left(P_{\mathrm{V}}-P_{\mathrm{at}}\right)+K_{\mathrm{iso}}\left(P_{\mathrm{ar}}-P_{\mathrm{at}}\right) & \text { for } P_{\mathrm{V}} \geq P_{\mathrm{ar}}\end{cases} \\
& R_{p} C \dot{P_{\mathrm{ar}}}+P_{\mathrm{ar}}-P_{\mathrm{ve}}=\left(R_{p}+Z_{c}\right) q+R_{p} Z_{c} C \dot{q}
\end{aligned}
$$

Table 7: Mechanical equations of the 0D model

$f= \begin{cases}\frac{t-T_{d, g l o b a l}}{Q R S_{\text {duration }}} & \text { when } T_{d, \text { global }} \leq t \leq T_{d, \text { global }}+Q R S_{\text {duration }} \\ 1 & \text { when } T_{d, \text { global }}+Q R S_{\text {duration }} \leq t \leq T_{r, \text { global }} \\ \left(1-\frac{t-T_{r, \text { global }}}{\left.Q R S_{\text {duration }}\right)}\right. & \text { when } T_{r, \text { global }} \leq t \leq T_{r, \text { global }}+Q R S_{\text {duration }} \\ 0 & \text { otherwise }\end{cases}$

$|u|_{+}=k_{\mathrm{ATP}} \cdot f_{\text {depo }}$

$|u|_{-}=k_{\mathrm{RS}} \cdot\left(1-f_{\text {depo }}\right)$

Table 8: Electrical activation in the 0D model

\section{References}

Baillargeon, B., Rebelo, N., Fox, D. D., Taylor, R. L., and Kuhl, E. (2014). The living heart project: a robust and integrative simulator for human heart function. European Journal of Mechanics-A/Solids, 48:38-47.

Caruel, M., Chabiniok, R., Moireau, P., Lecarpentier, Y., and Chapelle, D. (2014). Dimensional reductions of a cardiac model for effective validation and calibration. Biomechanics and modeling in mechanobiology, 13(4):897914.

Chabiniok, R., Moireau, P., Lesault, P.-F., Rahmouni, A., Deux, J.-F., and Chapelle, D. (2012). Estimation of tissue contractility from cardiac cine-mri using a biomechanical heart model. Biomechanics and modeling in mechanobiology, 11(5):609-630.

Chabiniok, R., Wang, V. Y., Hadjicharalambous, M., Asner, L., Lee, J., Sermesant, M., Kuhl, E., Young, A. A., Moireau, P., Nash, M. P., Chapelle, D., and Nordsletten, D. A. (2016). Multiphysics and multiscale modelling, data-model fusion and integration of organ physiology in the clinic: ventricular cardiac mechanics. Interface Focus, 6(2).

Chapelle, D., Le Tallec, P., Moireau, P., and Sorine, M. (2012). Energy-preserving muscle tissue model: formulation and compatible discretizations. International Journal for Multiscale Computational Engineering, 10(2).

Chen, Z., Cabrera-Lozoya, R., Relan, J., Sohal, M., Shetty, A., Karim, R., Delingette, H., Gill, J., Rhode, K., Ayache, N., Taggart, P., Rinaldi, C. A., Sermesant, M., and Razavi, R. (2016). Biophysical modelling predicts ventricular tachycardia inducibility and circuit morphology: A combined clinical validation and computer modelling approach. Journal of Cardiovascular Electrophysiology, 27(7):851-860

Clayton, R., Bernus, O., Cherry, E., Dierckx, H., Fenton, F., Mirabella, L., Panfilov, A., Sachse, F. B., Seemann, G., and Zhang, H. (2011). Models of cardiac tissue electrophysiology: progress, challenges and open questions. Progress in biophysics and molecular biology, 104(1):2248.

Cuellar, A. A., Lloyd, C. M., Nielsen, P. F., Bullivant, D. P., Nickerson, D. P., and Hunter, P. J. (2003). An overview 
of cellml 1.1, a biological model description language. SIMULATION, 79(12):740-747.

Duchateau, N., De Craene, M., Allain, P., Saloux, E., and Sermesant, M. (2016). Infarct localization from myocardial deformation: Prediction and uncertainty quantification by regression from a low-dimensional space. Transactions on Medical Imaging.

Garny, A. and Hunter, P. J. (2015). Opencor: a modular and interoperable approach to computational biology. Frontiers in Physiology, 6(26).

Geijtenbeek, T., van de Panne, M., and van der Stappen, A. F. (2013). Flexible muscle-based locomotion for bipedal creatures. ACM Transactions on Graphics (TOG), 32(6):206.

Hansen, N. (2006). The cma evolution strategy: a comparing review. In Towards a new evolutionary computation, pages 75-102. Springer Berlin Heidelberg.

Huxley, A. (1957). Muscle structure and theories of contraction. Progress in biophyics and biophysical chemistry, $7: 255-318$.

Jolly, M.-P., Guetter, C., Lu, X., Xue, H., and Guehring, J. (2011). Automatic segmentation of the myocardium in cine $\mathrm{mr}$ images using deformable registration. In International Workshop on Statistical Atlases and Computational Models of the Heart, pages 98-108. Springer.

Kayvanpour, E., Mansi, T., Sedaghat-Hamedani, F., Amr, A., Neumann, D., Georgescu, B., Seegerer, P., Kamen, A., Haas, J., Frese, K. S., Irawati, M., Wirsz, E., King, V., Buss, S., Mereles, D., Zitron, E., Keller, A., Katus, H. A., Comaniciu, D., and Meder, B. (2015). Towards Personalized Cardiology: Multi-Scale Modeling of the Failing Heart. PLoS ONE, 10(7).

Kennedy, M. C. and O'Hagan, A. (2000). Predicting the output from a complex computer code when fast approximations are available. Biometrika, 87(1):1-13.

Marchesseau, S., Delingette, H., Sermesant, M., and Ayache, N. (2013a). Fast parameter calibration of a cardiac electromechanical model from medical images based on the unscented transform. Biomechanics and modeling in mechanobiology, 12(4), 815-831.

Marchesseau, S., Delingette, H., Sermesant, M., Lozoya, R. C., Tobon-Gomez, C., Moireau, P., i Ventura, R. M. F., Lekadir, K., Hernández, A. I., Garreau, M., Donal, E., Leclercq, C., Duckett, S. G., Rhode, K. S., Rinaldi, C. A., Frangi, A. F., Razavi, R., Chapelle, D., and Ayache, N. (2013b). Personalization of a cardiac electromechanical model using reduced order unscented kalman filtering from regional volumes. Medical Image Analysis, 17(7):816-829.

Marchesseau, S., Heimann, T., Chatelin, S., Willinger, R., and Delingette, H. (2010). Multiplicative jacobian energy decomposition method for fast porous viscohyperelastic soft tissue model. In International Conference on Medical Image Computing and ComputerAssisted Intervention, pages 235-242. Springer.

Mollero, R., Pennec, X., Delingette, H., Ayache, N., and Sermesant, M. (2016). A multiscale cardiac model for fast personalisation and exploitation. In International Conference on Medical Image Computing and ComputerAssisted Intervention, pages 174-182. Springer.

Neumann, D., Mansi, T., Itu, L., Georgescu, B., Kayvanpour, E., Sedaghat-Hamedani, F., Amr, A., Haas, J., Katus, H., Meder, B., et al. (2016). A self-taught artificial agent for multi-physics computational model personalization. Medical image analysis, 34:52-64.

Panthee, N., Okada, J.-i., Washio, T., Mochizuki, Y., Suzuki, R., Koyama, H., Ono, M., Hisada, T., and Sugiura, S.
(2016). Tailor-made heart simulation predicts the effect of cardiac resynchronization therapy in a canine model of heart failure. Medical image analysis, 31:46-62.

Peherstorfer, B., Willcox, K., and Gunzburger, M. (2016). Survey of multifidelity methods in uncertainty propagation, inference, and optimization.

Powell, M. J. (2009). The bobyqa algorithm for bound constrained optimization without derivatives. Cambridge NA Report NA2009/06, University of Cambridge, Cambridge.

Schaerer, J., Qian, Z., Clarysse, P., Metaxas, D., Axel, L., and Magnin, I. E. (2006). Fast and automated creation of patient-specific $3 \mathrm{~d}$ heart model from tagged mri. In Proceedings of the MICCAI 2006 SA2PM Workshop.

Seegerer, P., Mansi, T., Jolly, M.-P., Neumann, D., Georgescu, B., Kamen, A., Kayvanpour, E., Amr, A., Sedaghat-Hamedani, F., Haas, J., et al. (2015). Estimation of regional electrical properties of the heart from 12-lead ECG and images. volume 8896 of LNCS.

Sermesant, M., Chabiniok, R., Chinchapatnam, P., Mansi, T., Billet, F., Moireau, P., Peyrat, J.-M., Wong, K. C., Relan, J., Rhode, K. S., Ginks, M., Lambiase, P., Delingette, H., Sorine, M., Rinaldi, C. A., Chapelle, D., Razavi, R., and Ayache, N. (2012). Patient-Specific Electromechanical Models of the Heart for Prediction of the Acute Effects of Pacing in CRT: a First Validation. Medical Image Analysis, 16(1):201-215.

Smith, N., de Vecchi, A., McCormick, M., Nordsletten, D., Camara, O., Frangi, A. F., Delingette, H., Sermesant, M., Relan, J., Ayache, N., Krueger, M. W., Schulze, W. H. W., Hose, R., Valverde, I., Beerbaum, P., Staicu, C., Siebes, M., Spaan, J., Hunter, P., Weese, J., Lehmann, H., Chapelle, D., and Rezavi, R. (2011). euheart: personalized and integrated cardiac care using patient-specific cardiovascular modelling. Interface Focus, 1(3):349-364.

Streeter, D. D. (1979). Gross morphology and fiber geometry of the heart. Handbook of physiology, pages 61-112.

Wang, V. Y., Hoogendoorn, C., Frangi, A. F., Cowan, B. R., Hunter, P. J., Young, A. A., and Nash, M. P. (2012). Automated personalised human left ventricular fe models to investigate heart failure mechanics. In International Workshop on Statistical Atlases and Computational Models of the Heart, pages 307-316. Springer.

Wang, Y., Georgescu, B., Chen, T., Wu, W., Wang, P., Lu, X., Ionasec, R., Zheng, Y., and Comaniciu, D. (2013). Learning-based detection and tracking in medical imaging: a probabilistic approach. In Deformation Models, pages 209-235. Springer.

Westerhof, N., Bosman, F., De Vries, C. J., and Noordergraaf, A. (1969). Analog studies of the human systemic arterial tree. Journal of biomechanics, 2(2).

Xi, J., Lamata, P., Lee, J., Moireau, P., Chapelle, D., and Smith, N. (2011). Myocardial transversely isotropic material parameter estimation from in-silico measurements based on a reduced-order unscented kalman filter. Journal of the mechanical behavior of biomedical materials, 4(7):1090-1102.

Yu, T., Lloyd, C. M., Nickerson, D. P., Cooling, M. T., Miller, A. K., Garny, A., Terkildsen, J. R., Lawson, J., Britten, R. D., Hunter, P. J., and Nielsen, P. M. F. (2011). The physiome model repository 2. Bioinformatics, 27(5):743. 